\title{
Fibre-Array Optical Interconnection for Silicon Photonics
}

T. Tekin (1), H. Schröder (1), L. Zimmermann (2), P. Dumon (3), W. Bogaerts (3)

1: Fraunhofer Institute for Reliability and Microintegration (IZM), G.-Meyer-Allee 25, 13355 Berlin, Germany; tolga.tekin@izm.fraunhofer.de

2: TU Berlin, HFT 4, Einsteinufer 25, 10587 Berlin, Germany, lars.zimmermann@tu-berlin.de

3: Ghent University-IMEC, INTEC, Sint-Pietersnieuwstr. 41, 9000 Gent, Belgium, pieter.dumon@intec.ugent.be

\begin{abstract}
Commercial multi-fibre array coupled to vertical grating coupler on a SOI (Silicon on insulator) chip, which is suitable for cost effective, compact interconnect for smart photonics packaging (ePIXpack).
\end{abstract}

\section{Introduction}

Silicon photonics is a rapidly advancing field with a strong potential for applications in integrated photonics. These applications comprise a wide spectrum ranging from optical sensors to optical data \& telecom sub-modules. The most outstanding merit of Silicon photonics is the use of highly advanced microelectronics process technologies. This allows for the fabrication of ultra-compact and low-loss Silicon waveguide devices (photonic nanowires) in a CMOScompatible fashion, opening the way for a true convergence of electronics and photonics.

\section{High-Index Grating Coupler}

Coupling to silicon photonic wires through high-index contrast gratings is attractive because of the relaxed alignment tolerances compared to facet coupling of standard single mode fibres. Because of the high index contrast, the grating can be short (25 periods) and achieve a relatively large bandwidth.

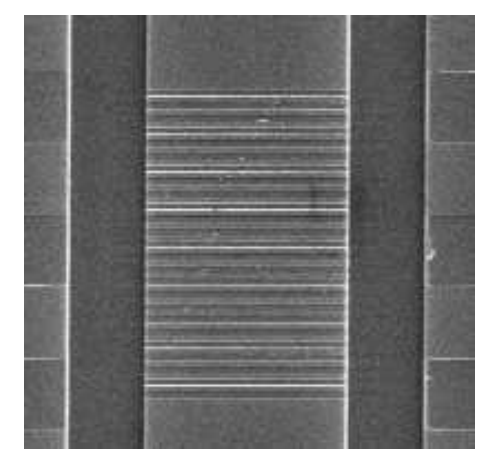

Figure 1: Uniform fibre couplers etched in a $10 \mu \mathrm{m}$ wide SOI waveguide.[1, 2]

Simple one-dimensional grating couplers with a uniform fill factor etched into a broad waveguide (Figure 1), achieve a coupling efficiency of around $30 \%$ with a $40 \mathrm{~nm} 1 \mathrm{~dB}$ bandwidth (per coupler) for a single polarization $[1,2]$. Detuned gratings with a coupling angle of $8^{\circ}$ to $10^{\circ}$ are used in order to avoid coupling to the wrong direction. The measured alignment tolerance for a $1 \mathrm{~dB}$ loss penalty is $\pm 2 \mu \mathrm{m}$. The grating couplers can be optimized in various ways to improve the efficiency or size.

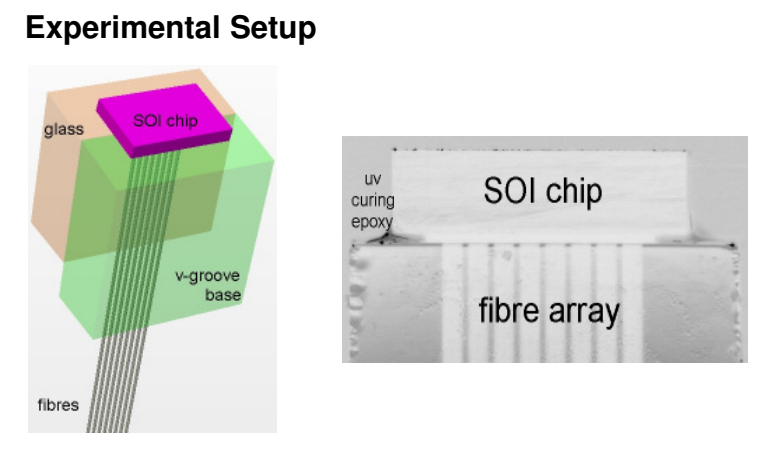

Figure 2: (a) Schematic and (b) picture of the fibre array interconnection.

For the demonstration of the first fibre array interconnection for silicon photonics, a SOI chip and a commercial fibre array have been used (Figure 2a-b). The SOI chip consists of 6 spare grating coupler ports, which are shortened by simple waveguides (Figure 3). The commercial fibre array as depicted in the Figure. $2 \mathrm{a}$ and consists of a v-groove base, a glass lid and 8 fibres, where the front facet polishing angle was $8^{\circ}$ (Figure 2a). The pigtailing of the array with FC/PC connectors was realized in our laboratories.

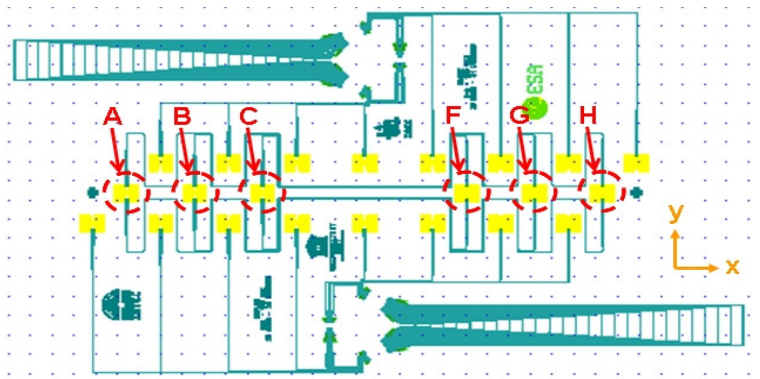

spare grating couplers shortened by simple waveguide

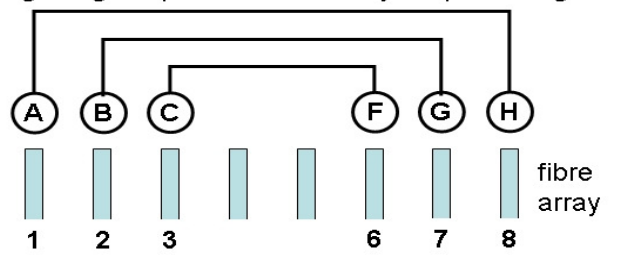

Figure 3: Layout of the SOI chip with shortened grating couplers and optical interconnection scheme using a commercial fibre array. 
To investigate the tolerances of the array, a CW light source (HP81553SM, 1550nm) was connected to one of the fibres of the array. By using a precise alignment system (PI F206) a probe fibre was coupled to the array to measure the optical power (HP81532A) while its position was changed. Such transmission measurement was performed for all six corresponding ports of the array. The alignment sensitivity of fibre array to the SOI chip was investigated where the position of the SOI chip was changed and the transmission through the ports $\mathrm{AH}, \mathrm{BG}$, and $\mathrm{CF}$ were measured, respectively.

After active alignment the position of the SOI chip was fixed using a UV-curing epoxy. The wavelength dependence of the transmission through shortened grating couplers was measured, where HP8168A was used as an ECL (external cavity laser).

\section{Results and Discussion}

To our knowledge this is the first demonstration of a thoroughly characterized fibre array interconnection for silicon photonics with commercial fibre array and SOI chip with grating couplers.

\begin{tabular}{|l|l|l|l|l|l|l|}
\hline fibre \# & 1 & 2 & 3 & 6 & 7 & 8 \\
\hline $\begin{array}{l}\text { mismatch } \\
\text { in } x[\mu \mathrm{m}]\end{array}$ & -2.2 & 0.8 & 0.3 & 0.1 & 0.0 & 2.0 \\
\hline $\begin{array}{l}\text { mismatch } \\
\text { in } y[\mu \mathrm{m}]\end{array}$ & 0.0 & 4.0 & 1.7 & -2.5 & -4.3 & 0.0 \\
\hline loss $[\mathrm{dB}]$ & 5.56 & 5.26 & 5.39 & 6.94 & 6.43 & 8.97 \\
\hline
\end{tabular}

Table 1: The measured tolerances within the multi fibre array with eight fibres.

The measured tolerances such as lateral displacement and transmission losses due to the pigtailing process of the array are summarized in table 1. Here the discrepancy relative to the ideal condition is due to the tolerances of the v-groove base which is the carrier for the fibres and also due to the core/cladding geometry of the fibres themselves.

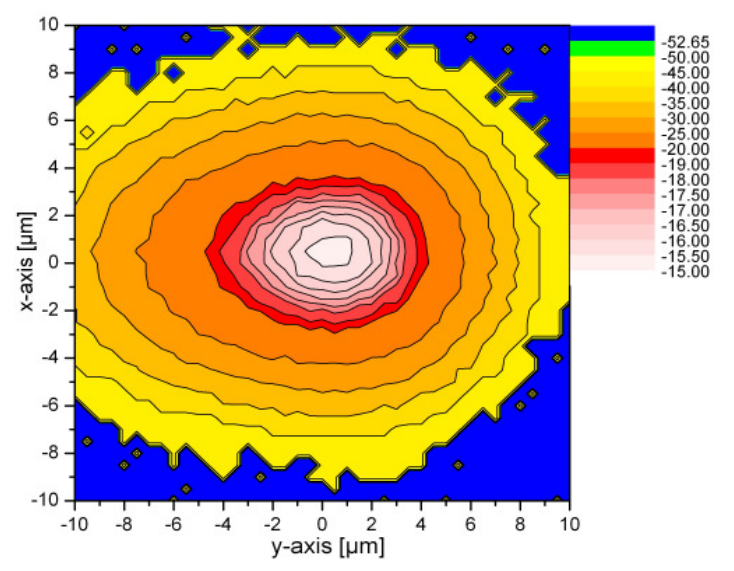

Figure 4: Measured fibre-to-fibre transmission showing lateral alignment sensitivity. Z-axis: waveguide propagation direction; $x$-axis: parallel to grating grooves; each $0.5 \mathrm{~dB}$ contour lines are shown
Typical alignment sensitivity is depicted in figure 4 . The measured sensitivity is summarized in the following table 2. Hereto, the transmission loss, $1 \mathrm{~dB}$ and $3 \mathrm{~dB}$ loss penalties in the lateral direction are provided.

\begin{tabular}{|l|c|c|c|}
\hline \multicolumn{1}{|c|}{ transmission } & $1 \mathrm{AH} 8$ & 2BG7 & 3CF6 \\
\hline loss [dB] & 16.48 & 15.18 & 12.56 \\
\hline $\begin{array}{l}1 \mathrm{~dB} \text { loss penalty } \\
\text { at x-axis [ } \mu \mathrm{m}]\end{array}$ & \pm 1 & \pm 1 & \pm 1.5 \\
\hline $\begin{array}{l}1 \mathrm{~dB} \text { loss penalty } \\
\text { at y-axis [ } \mu \mathrm{m}]\end{array}$ & \pm 2 & \pm 1.5 & \pm 2 \\
\hline $\begin{array}{l}\text { 3dB loss penalty } \\
\text { at x-axis [ } \mu \mathrm{m}]\end{array}$ & \pm 2.5 & \pm 2.5 & \pm 2.8 \\
\hline $\begin{array}{l}\text { 3dB loss penalty } \\
\text { at y-axis [ } \mu \mathrm{m}]\end{array}$ & \pm 3.5 & \pm 3.5 & \pm 3.5 \\
\hline
\end{tabular}

Table 2: Summary of alignment sensitivity.

The wavelength dependence of transmission through the shortened grating couplers is depicted in Figure 5.

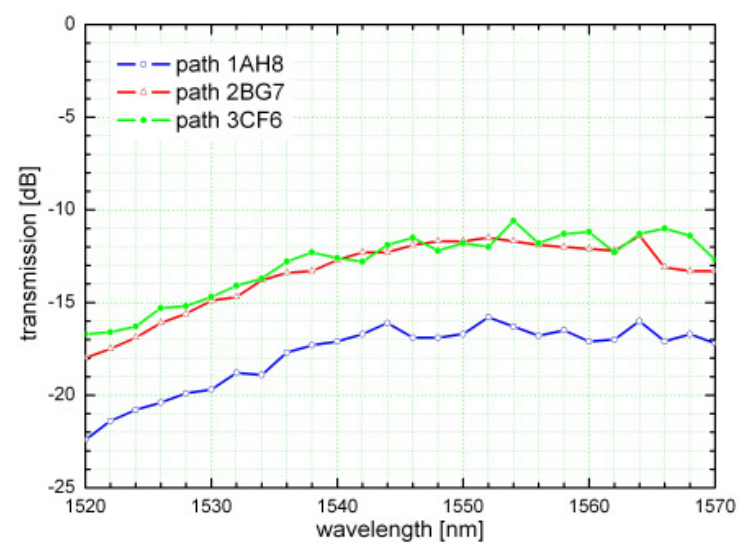

Figure 5: Wavelength dependence of transmission through the shortened grating couplers.

\section{Conclusions}

We have demonstrated the first fully characterized fibre array interconnection for silicon photonics with commercial fibre array and SOI chip using grating couplers. The tolerances of the used fibre array and the alignment sensitivity of the SOI chip were analyzed, respectively. The demonstrated interconnection has an enormous potential as a smart, low cost, compact package for silicon photonics.

\section{Acknowledgements}

This article is the result of cooperation in the frame of the Network of Excellence ePIXnet, the ePIXnet Silicon Photonics Platform and of the Packaging Platform ePIXpack.

\section{References}

1. D. Taillaert et al Ja J Ap Phy $\underline{45}$ p. 6071 (2006)

2. P. Dumon et al Optics. Express. $664 \underline{14} 2$ (2006) 